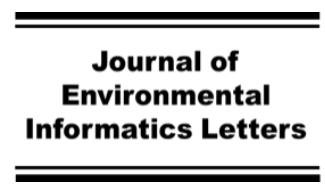

Www.iseis.org/jeil

\title{
Prediction of Long-Term Near-Surface Temperature Based on NA-CORDEX Output
}

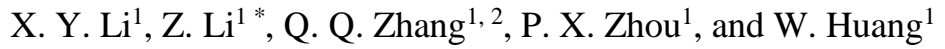 \\ ${ }^{I}$ Department of Civil Engineering, McMaster University, Hamilton, Ontario L8S 4L7, Canada \\ ${ }^{2}$ School of Management, Chengdu University of Information Technology, Chengdu 610225, China
}

Received 1 May 2019; revised 9 June 2019; accepted 18 July 2019; published online 12 August 2019

\begin{abstract}
Temperature is one of the most important parameters in climate modeling, as it has significant impacts on various geophysical processes such as evaporation and precipitation. Applying multiple climate models for prediction generally outperforms the use of individual climate models, and neural networks perform well at capturing nonlinear relationships, which can provide more reliable temperature projections. In this study, three neural network algorithms, including Multi-layer Perceptron (MLP), Time-lagged Feed-forward Neural Networks (TLFN) and Nonlinear Auto-Regressive Networks with exogenous inputs (NARX), were used to develop data-driven models for predicting daily mean near-surface temperature based on North American Coordinated Regional Downscaling Experiment (NA-CORDEX) output. A case study of Big Trout Lake in Ontario, Canada was carried out to demonstrate the applications and to evaluate the performance of the proposed neural network based models. The results showed that MLP, TLFN, and NARX performed well in generating accurate daily near-surface temperature predictions with the coefficient of determination $\left(\mathrm{R}^{2}\right)$ values above 0.84 . The three neural network based models had similar performance with no significant difference in terms of root mean square error and $\mathrm{R}^{2}$. Neural network based climate prediction models outperformed each of the individual regional climate models and generated smoother predicttions with less fluctuation. This study provides a technical basis for generating reliable predictions of daily temperature using neural networks based model.
\end{abstract}

Keywords: Neural Networks, temperature prediction, Regional Climate Model, NA-CORDEX, Ontario

\section{Introduction}

Temperature changes have significant impacts on natural processes and human activities (Karl et al., 2009), for instance, biological changes (Parmesan and Yohe, 2003) and construction sensibility (Xia et al., 2012). Thus, predicting temperature precisely is of vital importance. Multiple climate models, such as Global Climate Model (GCM) and Regional Climate Model (RCM), have been developed and can be applied to temperature simulations and predictions, which provide support for climate impact analysis (Thomson et al., 2006; Li et al., 2016; Wagner et al., 2017). These models were developed by different institutions and their temperature predictions are not always consistent with one another. Although these models have errors in certain processes (e.g., cloud formation), they can provide plausible estimations for future variations in climate (Huo and $\mathrm{Li}$, 2012; Ragone et al., 2015).

Applying dynamic downscaling to drive RCM is computationally costly and time-consuming (Spak et al., 2007). Moreover, the uncertainties in the modeling system lead to an in-

\footnotetext{
* Corresponding author. Tel.: +1 905 5259140; fax: +1 9055727944.

E-mail address: zoeli@mcmaster.ca (Z. Li).

ISSN: $1726-2135$ print/1684-8799 online

(C) 2019 ISEIS All rights reserved. doi:10.3808/jeil.201900012
}

crease in forecast errors with increasing forecast length (Kumar et al., 2012). Using statistical methods to post-process multiple RCMs would help to better generate predictions with higher accuracy than an individual RCM (Palmer et al., 2005; Barfus and Bernhofer, 2014). Samouly (2018) applied mean and median values of multi-model ensembles for monthly temperature predictions, which showed better prediction performance than using a single RCM. However, as each model generates a different range of predictions and errors, the mean value calculated by allocating the same weight to each RCM may not be enough to fully take advantage of each prediction model.

Artificial neural networks (ANNs), which are more powerful than the regression-based techniques, have been widely applied in climate prediction because of their high potential for complex, nonlinear and time-varying input-output mapping (Von Storch et al., 2000). For instance, ANNs have been widely applied in statistical downscaling for temperature and precipitation prediction (Wilby and Wigley, 1997; Wilby et al., 1998). Previous studies suggest that using computer-based learning algorithms, such as ANNs, to develop accurate prediction models can profoundly reduce the long-term dependency (Siegelmann, 1997; Sfetsos, 2000; Shen and Chang, 2013; Caswell, 2014). Moreover, current and future temperatures have a close connection with the temperatures of previous days. Incorpo- 
rating both concurrent and antecedent predictor values as input could improve the accuracy of temperature prediction (Coulibaly et al., 2005). Various types of neural networks have an internal memory structures that can store information about past variables. Time-lagged feed-forward networks (TLFNs) and recurrent neural networks (RNNs) are the two major groups of dynamic neural networks that are commonly used in time series analysis (Coulibaly et al., 2001; Dibike and Coulibaly, 2006). A TLFN simply replaces the neurons in the input layer of a Multi-layer perception (MLP) with a memory structure. It is less complex than the RNNs and has similar capability for processing temporal patterns (Dibike et al., 1999). TLFN is an efficient method for downscaling both daily precipitation as well as daily maximum and minimum temperature series (Coulibaly et al., 2005). The Nonlinear Auto-Regressive Networks with exogenous inputs (NARX) model is a dynamic network that has been widely used for time series prediction (Dhussa et al., 2014). It can learn the behavior of a system in an effective way. It also converges much faster and generalizes better than other networks (Lin et al., 1996; Çoruh et al., 2014). It has been demonstrated that NARX is capable of capturing the dynamics of nonlinear complex systems (Diaconescu, 2008; Chan et al., 2015). Moreover, NARX performs favorably on long-term dependencies (Rahimi et al., 2018). Thus, NARX is particularly useful for time series modeling.

Considering that neural networks perform well at grasping the nonlinear relationships between predictors and predictands, MLP, TLFN, and NARX models will be applied to simulate daily mean near-surface temperature and generate predictions basing on multiple RCMs. The goal of this study is to develop, validate and evaluate the performance of neural networks for daily mean near-surface prediction with multiple RCMs in the province of Ontario, Canada. This entails the following: (1) collecting North American Coordinated Regional Downscaling Experiment (NA-CORDEX) data to provide inputs for the proposed neural network based models; (2) developing MLP, TLFN, and NARX models to generate daily mean near-surface temperature; (3) evaluating the performance of MLP, TLFN and NARX using a case study of the Big Trout Lake station in Ontario, Canada.

\section{Methodology}

\subsection{Multi-layer Perceptron (MLP)}

MLP is a widely-used ANN model which usually consists of an input layer, one or more hidden layers, and an output layer (Figure 1). Each layer includes some neurons (Jiang et al., 2018). The numbers of neurons in the input and output layers are determined by the numbers of elements in the external input array and output array of the network, respectively (Osman and Abdellatif, 2016). The number of neurons in the hidden layers are determined by the trial and error (Hammerstorm, 1993) for the best performing model. Different layers are connected with weights and biases. The connections between the layers allow information flow forward towards the output layer. The neuron network first computes the weighted sum of the inputs, $z$, and feeds $z$ into the neurons in the hidden layer (Equation (1)). A nonlinear activation function $f($.$) , is applied to z$ to get the output $a$ of the neuron (Equation (2)). The network repeats the same process to the hidden layer (Equations (3) and (4)). Rectified linear unit (ReLU), conventional sigmoids function, hyperbolic tangent function, and logistic function are examples of commonly used activation functions. The ultimate goal of training a MLP is to minimize the cost function (Equation (5)), which measures the errors between observations and predictions for training data. A back-propagation algorithm is used to find the minimum cost function using the chain rule of differentiation to calculate the partial derivative or gradient of the cost corresponding to the weights (Zhang et al., 2018). Backpropagation calculates the error-derivative for the weight of each neuron to minimize the cost function:

$$
\begin{aligned}
& z_{j}=\sum_{i}^{n} w_{i j} x_{i}+b_{j} \\
& a_{j}=f\left(z_{j}\right) \\
& z_{k}=\sum_{j}^{m} w_{j k} a_{j}+b_{k} \\
& \mathrm{y}_{k}=f\left(z_{k}\right) \\
& C_{k}=\frac{1}{2}\left(y_{k}-t_{k}\right)^{2} \\
& \frac{\partial C}{\partial z_{k}}=\frac{\partial C}{\partial y_{k}} \times \frac{\partial y_{k}}{\partial z_{k}} \\
& \frac{\partial C}{\partial y_{k}}=y_{k}-t_{k}
\end{aligned}
$$

where $x_{i}$ is the $i^{\text {th }}$ input, $a_{j}$ is the output of the $j^{\text {th }}$ neuron; $w_{i j}$ and $w_{j k}$ represent the weight of $j^{\text {th }}$ neuron in the hidden layer and $k^{\text {th }}$ neuron in the output layer, respectively; and $b$ is the bias. $C$ refers to the cost of the cost function, $y_{k}$ is the predicted output and $t_{k}$ is the observed true value. The error-derivative for the weight $w_{j k}$ on the connection from unit $k$ is $a_{j}(\partial C) /\left(\partial z_{k}\right)$. The error-derivative for the weight $w_{i j}$ on the connection from unit $j$ is $x_{i}(\partial C) /\left(\partial z_{j}\right)$ (Equation (6)) (LeCun et al., 2015). Equation (7) shows the partial derivative of the cost function corresponding and activation function.

\subsection{Time-lagged Feed-Forward Neural Network (TLFN)}

TLFN is formulated based on MLP and replaces the neurons in the input layer with a memory structure, which is sometimes called a tap delay-line, as shown in Figure 2 (Coulibaly et al., 2005). TLFN uses delay-line processing elements (PEs) by holding past samples of the input signal. The output $y(n)$ of TLFN with one hidden layer is shown as Equation (8) (Coulibaly, 2004): 


$$
\begin{aligned}
& y(n)=f_{1}\left(\sum_{j=1}^{m} w_{j} y_{j}(n)+b_{o}\right) \\
& =f_{1}\left\{\sum_{j=1}^{m} w_{j} f_{2}\left[\sum_{l=0}^{k} w_{j l} x(n-l)+b_{j}\right]+b_{o}\right\}
\end{aligned}
$$

where $m$ is the size of the hidden layer, $n$ is the time step, $w_{j}$ is the weight vector for the connection between the output layer and the hidden layer, and $w_{j l}$ is the weight matrix for the connection between the hidden layer and the input layer. $f_{1}$ and $f_{2}$ are the active functions at the output layer and hidden layer, respectively. $b_{o}$ and $b_{j}$ are the bias terms. The input pattern $x(n)$ has multiple inputs of size $p$ (Equation (9)) and $X(n)$ is the combined input at time step $n$, whose delay line with memory depth $k$ (Equation (10)). $x(n-1)$ is obtained by delaying $x(n)$ by one sample:

$$
\begin{aligned}
& x(n)=\left(x_{1}(n), x_{2}(n), \ldots, x_{p}(n)\right) \\
& X(n)=[x(n), x(n-1), \ldots, x(n-k+1)]
\end{aligned}
$$

$\begin{array}{ccc}\text { Input } & \text { Hidden } & \text { Output } \\ \text { Layer } & \text { Layer } & \text { Layer }\end{array}$

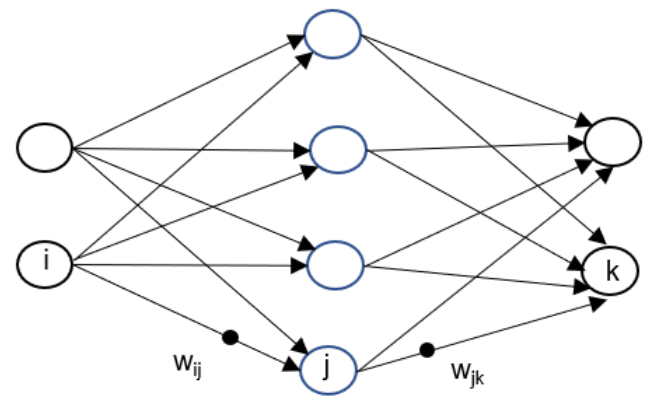

Figure 1. Structure of MLP with one input layer, one hidden layer(s), and one output layer.

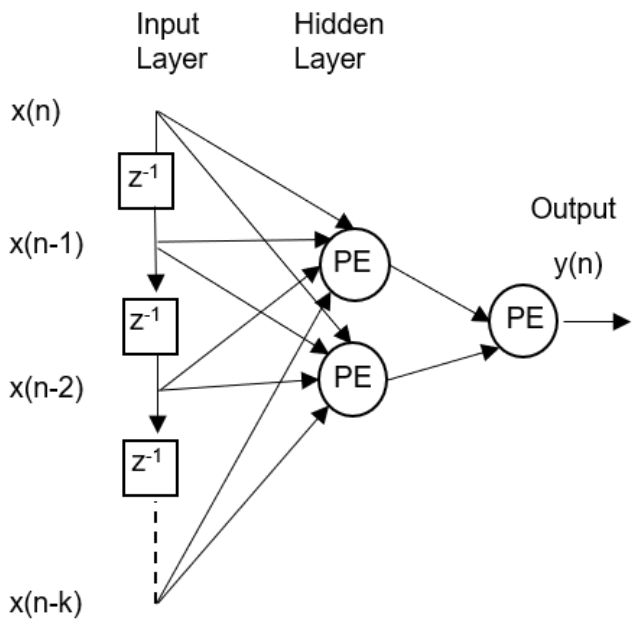

Figure 2. Structure of TLFN with one input layer, one hidden layer, and a delay-line with memory depth of $k$. $\left(z^{-1}\right.$ is an operator that delays the input by one sample). (Dibike and Coulibaly, 2006).

\subsection{Nonlinear Auto-Regressive Networks with Exogenous Inputs (NARX)}

Networks that use feedback connections, enabling information flow laterally or backwards within the network, are called RNNs. NARX is a special type of RNN that creates a relationship between the current value of a time series and the predicted past values of the exogenous series, and the outputs are fed back to the input by a delay line (Haykin, 1998). As shown in Figure 3, the structure of the NARX model is similar to the traditional multi-layered perceptron (MLP) model. The NARX model can be expressed as in Equation (11) (Lin et al., 1996):

$y_{t}=f\left(y_{t-1}, \ldots, y_{t-d y} ; x_{t}, x_{t-1}, \ldots, x_{t-d x}\right)$

where $x_{t}$ and $y_{t}$ represent the input and output of the network at time $t$, and $f($.$) is a nonlinear function, which can be approxi-$ mated by a standard MLP network. $d x$ and $d y$ are the time lags for the input and output series (Lin et al., 1998).

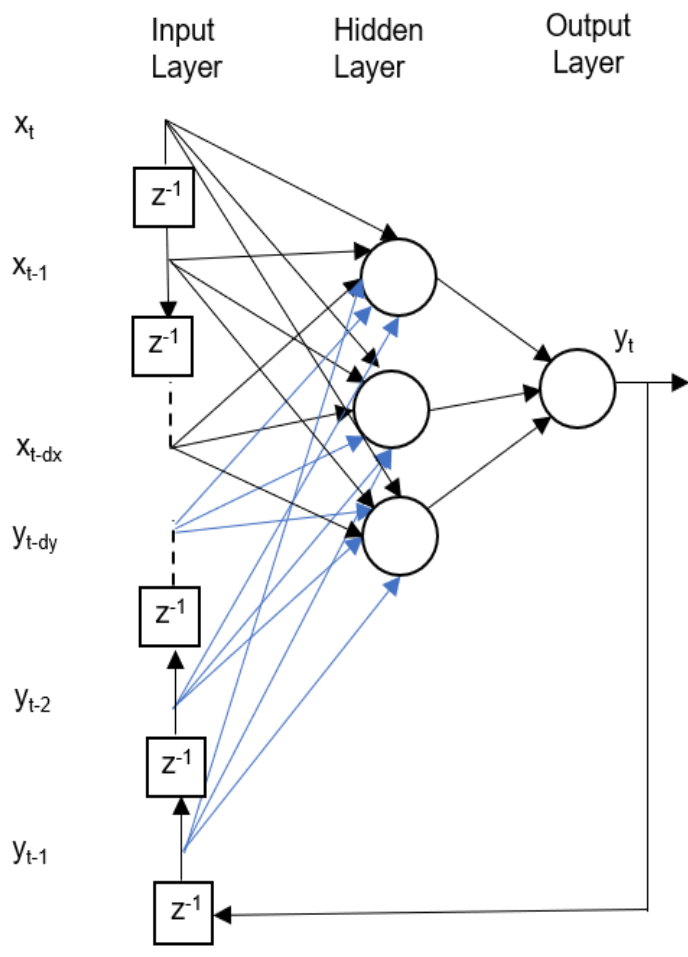

Figure 3. Structure of NARX network with one input layer, one hidden layer, and one output layer $\left(z^{-1}\right.$ denotes delay for one time step).

\section{Study Area and Data Collection}

Big Trout Lake in Northern Ontario, Canada was chosen 
to test the performance of the proposed methods. According to Canada's Changing Climate Report 2019 (Bush et al., 2019), Northern Canada has warmed and will continue to warm at even more than double the global rate. Between 1948 and 2016, the observed changes $\left({ }^{\circ} \mathrm{C}\right)$ in annual temperature in Northern Ontario were higher than in Southern. The Big Trout Lake station $\left(53.83^{\circ} \mathrm{N}, 89.87^{\circ} \mathrm{W}\right)$ is located in the far northwestern region of Ontario and south of Hudson Bay. It is classified as having a subarctic climate, which includes year-round precipitation, short and cool summers, and long and cold dry winters (Tam et al., 2018), resulting in high annual variation in temperature. The average temperature and yearly precipitation of Big Trout Lake are $-2.7{ }^{\circ} \mathrm{C}$ and $609.1 \mathrm{~mm}$. The average monthly temperature ranges from -23.7 to $16.2{ }^{\circ} \mathrm{C}$. The minimum and maximum recorded temperature of the Big Trout Lake station were $-47.8{ }^{\circ} \mathrm{C}$ (January 1951) and $35.6{ }^{\circ} \mathrm{C}$ (July 1955). The highest historical daily precipitation occurred in August 1955, reaching $84.1 \mathrm{~mm}$. Considering the region's high variation in temperature and climate sensitivity, the Big Trout Lake station was chosen for evaluating the performance of neural networks methods.

The study used daily mean temperature simulation data obtained from six RCMs and observation data of the Big Trout Lake station from 1979 to 1989 . The six RCMs are each driven by different GCM models. They are 1) CanRCM4, CRCM5, and RCA4 driven by CanESM2; 2) HIRHAM5 and RCA4 driven by EC-EARTH. 3) CRCM5 driven by MPI-ESM-LR. The grid resolution for each RCM is $0.44^{\circ} \times 0.44^{\circ}$. The simulated daily mean temperature data were downloaded from NA-CORDEX archive (Mearns et al., 2017), a branch of the International CORDEX Initiative (Giorgi et al., 2009; Lucas-Picher et al., 2012). The observed temperature data of the Big Trout Lake were down-loaded from the Digital Archive of Canadian Climatological Data provided by Environment and Climate Change Canada (ECCC).

\section{Neural Network Design and Training}

The neural network models in this study were developed with net functions in MATLAB (version R2014b). The Levenberg-Marquardt backpropagation algorithm was applied for training the models, as it is one of the fastest back-propagation algorithms for feedforward networks (Hagan and Menhaj, 1994; Lee et al., 2016).

Inputs to the neural networks were the simulated daily mean temperature of six RCMs while the output was daily mean near-surface temperature observed at the Big Trout Lake station. RCM outputs at the closest grid point to the Big Trout Lake station $\left(53.76^{\circ} \mathrm{N}, 89.84^{\circ} \mathrm{W}\right)$ were used as inputs for the pre-diction models. The first $70 \%$ of the dataset (January 1979 September 1986) were used for training the models. Then, the following $15 \%$ of the dataset (September $1986 \sim$ May 1988) were used to validate those models, which verified the applicability of the model. The last $15 \%$ of the dataset (May 1988 December 1989) were used for testing, which assessed the generalization ability of the model. The differrent parameters of each model were adjusted during calibration to obtain the best statistical agreement between observed and simulated mean temperature and were assessed using mean square error (MSE).

The structure of the networks used in the study consisted of one input layer, one output layer, and one hidden layer. MLP was trained with the number of neurons ranging from 5 to 20 and the MLP with 12 neurons was selected as it generated the best performing network. Both TLFN and NARX were trained with lag time (time delay) ranging from 1 to 3 days and the number of neurons ranging from 5 to 20 . The TLFN model with 5 neurons and a time lag of 3 days and NARX with 15 neurons and a time lag of 3 days were selected as they generated the best performing network.

Performance of three neural network models was evaluated by comparing predicted results with observed temperature values. Statistical criterions, such as root mean square error (RMSE) and coefficient of determination $\left(\mathrm{R}^{2}\right)$, were used for performance evaluation.

\section{Results and Discussion}

\subsection{Neural Networks Performance}

\subsubsection{MLP Performance}

The time-series plot and the scatter plot of the observation and prediction of daily mean temperature obtained by MLP are shown in Figures 4 and 5, respectively. The time-series plot shows that MLP could predict the seasonal pattern of daily mean near-surface temperature. RMSE and $\mathrm{R}^{2}$ of testing were $6.537^{\circ} \mathrm{C}$ and 0.843 , respectively. The small RMSE and high $\mathrm{R}^{2}$ values indicate that MLP performed well and could predict mean near-surface temperature with relatively high accuracy.

In addition, while the observed temperature of all datasets varied from -38.9 to $26.1{ }^{\circ} \mathrm{C}$, MLP could generate predictions ranging from -25.6 to $17.5^{\circ} \mathrm{C}$. For observations ranging from 30 to $-16{ }^{\circ} \mathrm{C}$, MLP tended to give prediction values of around $20{ }^{\circ} \mathrm{C}$. For observations ranging from 12 to $26.1^{\circ} \mathrm{C}$, MLP generated prediction ranging from 10 to $17^{\circ} \mathrm{C}$, which implies that MLP could not capture the extreme values precisely. This may be due to the tendency of neural networks sacrificing variance to gain high RMSE.

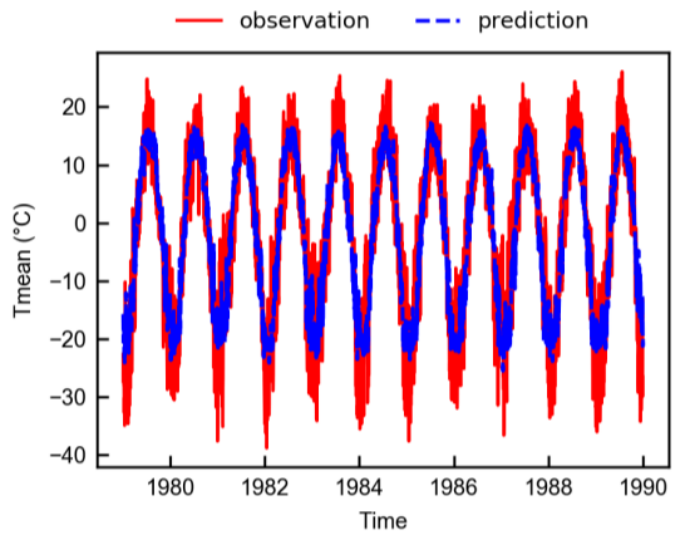

Figure 4. Time series plot of observed and predicted daily nearsurface temperature values obtained by MLP with 12 neurons. 
(a) training

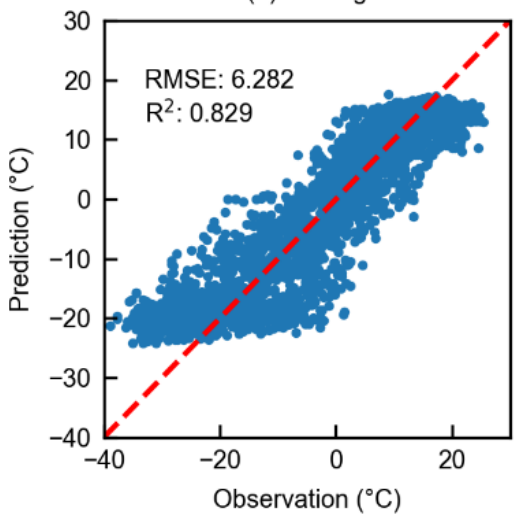

(b) validation

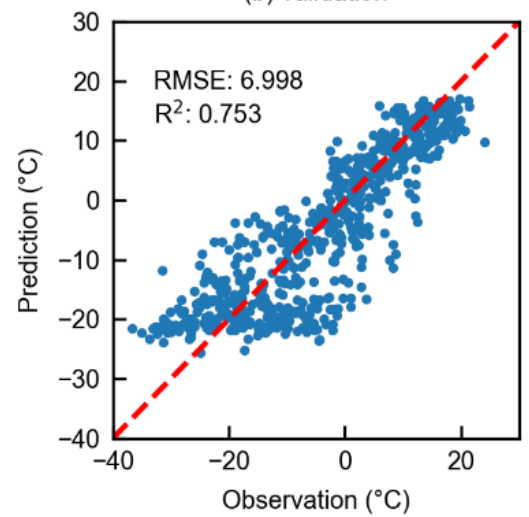

(c) testing

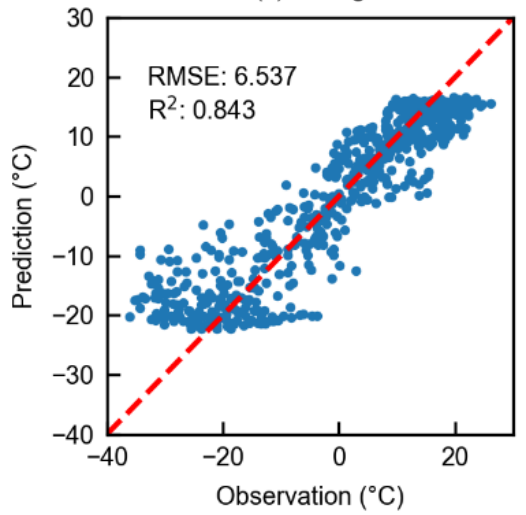

Figure 5. Scatter plots of observed and predicted daily near-surface temperature values obtained by MLP with 12 neurons.

Table 1. Comparison of Performance between RCMs and Neural Networks

\begin{tabular}{llllllll}
\hline & & \multicolumn{3}{c}{ RMSE $\left({ }^{\circ} \mathrm{C}\right)$} & \multicolumn{3}{c}{$\mathrm{R}^{2}$} \\
\cline { 3 - 7 } GCM & RCM & training & validation & testing & training & validation & testing \\
\hline CanESM2 & CRCM5 & 8.682 & 8.800 & 8.861 & 0.717 & 0.638 & 0.728 \\
& CanRCM4 & 9.402 & 9.124 & 9.239 & 0.665 & 0.617 & 0.691 \\
& RCA4 & 9.029 & 8.709 & 9.008 & 0.690 & 0.653 & 0.708 \\
EC-EARTH & HIRHAM5 & 8.349 & 8.411 & 8.547 & 0.695 & 0.659 & 0.722 \\
& RCA4 & 9.304 & 10.475 & 8.676 & 0.667 & 0.565 & 0.707 \\
MPI-ESM-LR & CRCM5 & 8.321 & 9.406 & 8.253 & 0.705 & 0.612 & 0.732 \\
Neural Networks & MLP & 6.282 & 6.998 & 6.537 & 0.829 & 0.753 & 0.843 \\
& TLFN & 6.244 & 6.797 & 6.363 & 0.832 & 0.766 & 0.854 \\
& NARX & 5.966 & 6.882 & 6.345 & 0.849 & 0.761 & 0.856 \\
\hline
\end{tabular}

\subsubsection{TLFN Performance}

Figures 6 and 7 show the statistical performance and time series plot of TLFN with a time lag of 3 days and 5 neurons. TLFN had similar performance with MLP and had small improvement, with RMSE decreased to $6.363{ }^{\circ} \mathrm{C}$ and $\mathrm{R}^{2}$ increased to 0.854 . This indicates that TLFN is an efficient model for capturing the changing pattern and predicting daily mean nearsurface temperature. Compared with the MLP model, TLFN generated predictions scattered more closely with observations and had a smaller range of temperature prediction from -23.9 to $16.5{ }^{\circ} \mathrm{C}$. For observations ranging from -30 to $-20{ }^{\circ} \mathrm{C}$, the over-estimated prediction errors of TLFN were smaller than MLP. Similar to MLP, TLFN did not capture the extreme values well.

\subsubsection{NARX Performance}

Figures 8 and 9 show that NARX performs well at generating mean temperature prediction and could accurately predict the changes of daily mean near-surface temperaturewith a low RMSE of $6.345^{\circ} \mathrm{C}$ and high $\mathrm{R}^{2}$ of 0.856 . This suggests that NARX performs the most effectively among the three in predicting daily mean near-surface temperature. The prediction range generated by NARX was from -25.4 to $15.7^{\circ} \mathrm{C}$; the maximum value was lower than the predictions generated by MLP and TLFN. From the scatter plot of observed and predicted tem- peratures shown in Figure 9, the points are scattered more densely along the diagonal line than MLP and TLFN, indicating that the error of prediction and observation values were smaller than that of MLP and TLFN. However, the accuracy of NARX for prediction extreme temperature values was similar to MLP and TLFN.

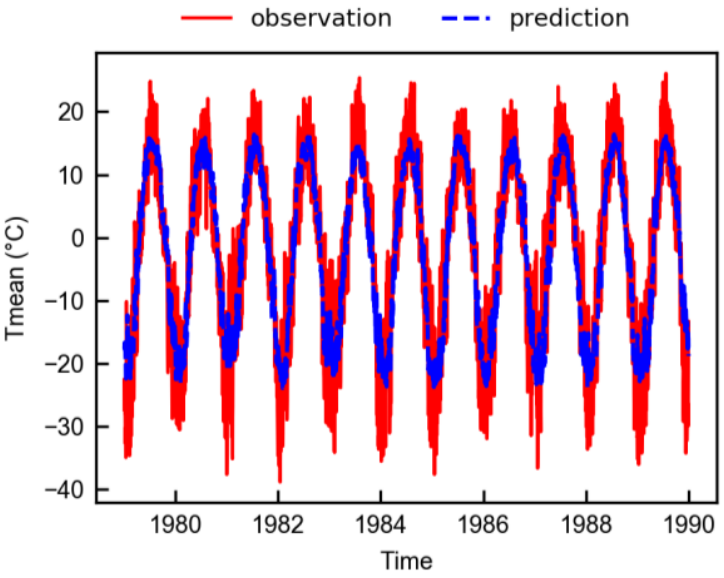

Figure 6. Time series plot of observed and predicted daily nearsurface temperature values obtained by TLFN with 5 neurons and a time lag of 3 days. 
(a) training

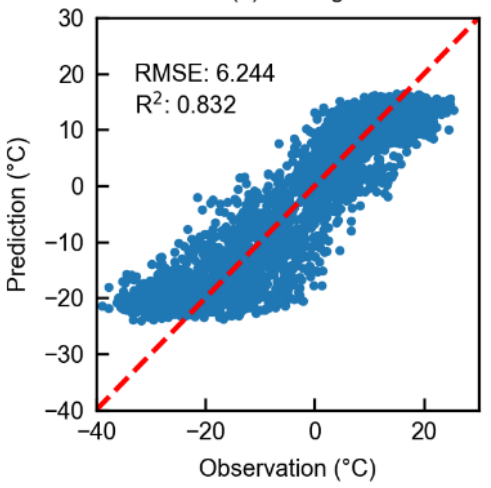

(b) validation

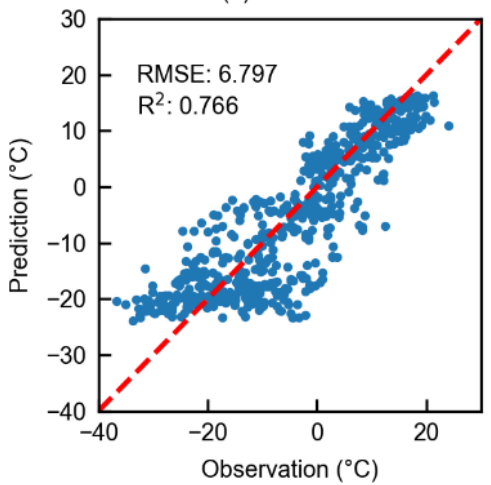

(c) testing

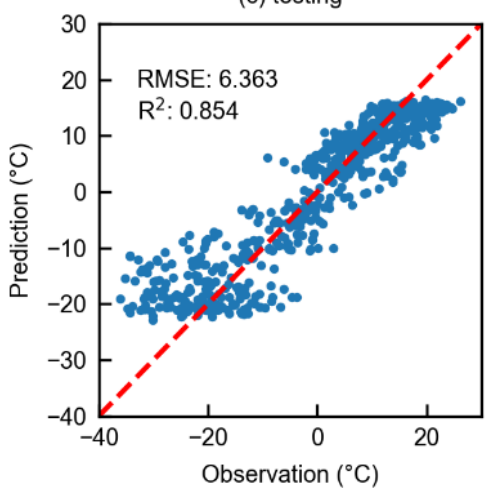

Figure 7. Scatter plots of observed and predicted daily near-surface temperature values obtained by TLFN with 5 neurons and a time lag of 3 days.

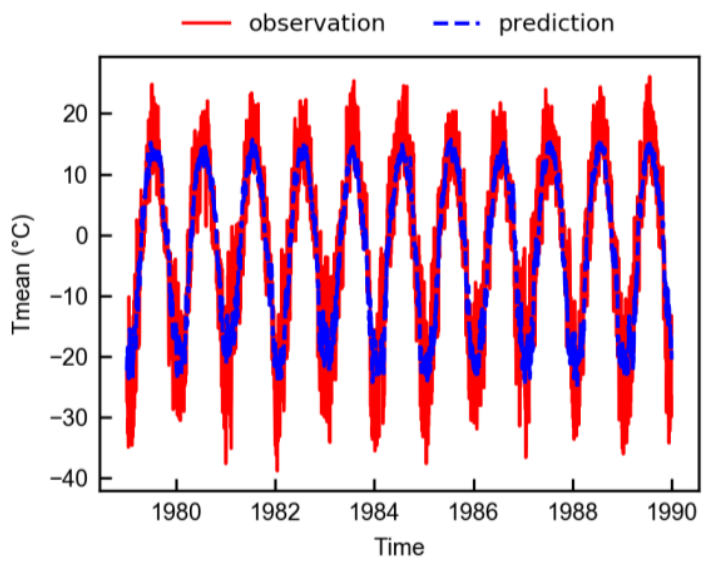

Figure 8. Time series plot of observed and predicted daily nearsurface temperature values obtained by NARX with 15 neurons and a time lag of 3 days.

\subsection{Comparison between RCMs and Neural Networks}

Table 1 shows the statistical performance of six RCMs and 3 neural network models for training, validation, and testing. The RMSE of six models ranged from 8.253 to $9.239{ }^{\circ} \mathrm{C}$ and the $\mathrm{R}^{2}$ ranged from 0.691 to 0.732 . Among the six models, CRCM5 derived by MPI_ESM_LR performed the best while CanRCM4 derived by CanESM 2 had the lowest $\mathrm{R}^{2}$ and the highest RMSE. Neural network based models outperformed each individual RCM model, with RMSE decreased by approximately $2{ }^{\circ} \mathrm{C}$ and $\mathrm{R}^{2}$ increased from 0.7 to 0.85 .

Figure 10 shows the time series plot of six RCMs and neural networks of winter (January and February) and summer (July and August) 1989 predictions. The observation values fall within the range of the RCMs, while the neural networks tended to predict temperatures of -20 and $15{ }^{\circ} \mathrm{C}$ for winter and summer, respectively, with very little variance and fluctuation. For winter, three RCMs driven by Can-ESM2 predicted relatively well with observations aligning closely to the RCMs predicted values. RCA4 driven by EC-EARTH performed the worst which tended to overestimate the low temperature and underestimated the high temperature in winter. CRCM5 driven by MPI-ESM-LR performed well with low error in winter. All RCMs performed better in the summer months than in the winter months, with observation values falling between the maximum and minimum prediction of 6 RCMs. However, the changing pattern of neural network based models was smoother than RCMs with small fluctuation. As neural network based models generate predictions with smooth variation pattern, they tend to have smaller RMSE and higher $\mathrm{R}^{2}$ than RCMs.

\subsection{Comparison of Performance between MLP, TLFN, and NARX}

The RMSE for all three methods ranged from 6.345 to $6.537{ }^{\circ} \mathrm{C}$ and $\mathrm{R}^{2}$ were above 0.84 , indicating that the three neural networks could provide reliable temperature forecasts for Big Trout Lake. The nonlinear transfer function associated with each hidden and output node allows ANNs to approximate highly nonlinear relationships without a prior assumption, which leads to relatively high accuracy in prediction.

In terms of the structure of neural networks, TLFN and NARX were built based on the structure of MLP. These two methods incorporate antecedent predictor values as input to improve forecasting. Although all three methods had similar performance with no significant differences in terms of RMSE and $\mathrm{R}^{2}$, TLFN and NARX had a smaller error in prediction than MLP. Thus, incorporating antecedent predictor values as input would slightly improve the performance of the neural network. When compared with TLFN, NARX not only incorporates previous RCM data into the network but also considers previously predicted values. However, the time required to train the NARX model and generate predictions was much longer than that of TLFN. As TLFN has similar capability to process and predict temporal patterns as RNN while having a less complex structure being less computationally demanding, TLFN is recommended for the prediction of temperature values in areas where the climate is similar to the study area. This finding is consistent with the results from previous studies on using neural networks for temperature predictions (Coulibaly et al., 2001; Coulibaly et al., 2005). 

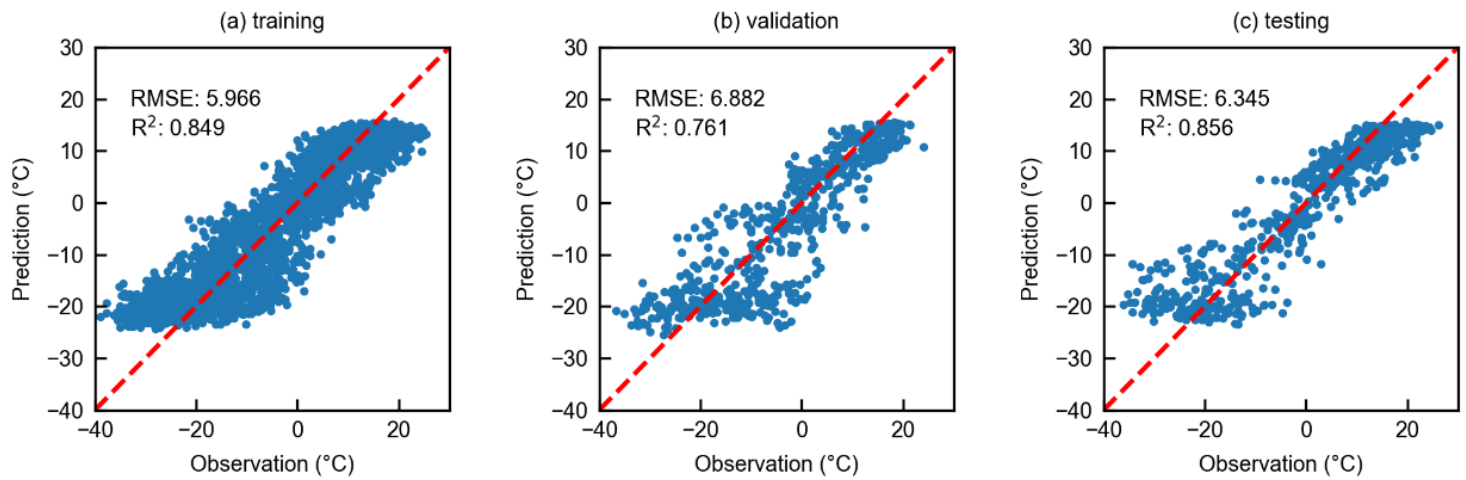

Figure 9. Scatter plot of observed and predicted daily near-surface temperature values obtained by NARX with 15 neurons and a time lag of 3 days.

(a) RCM models winter

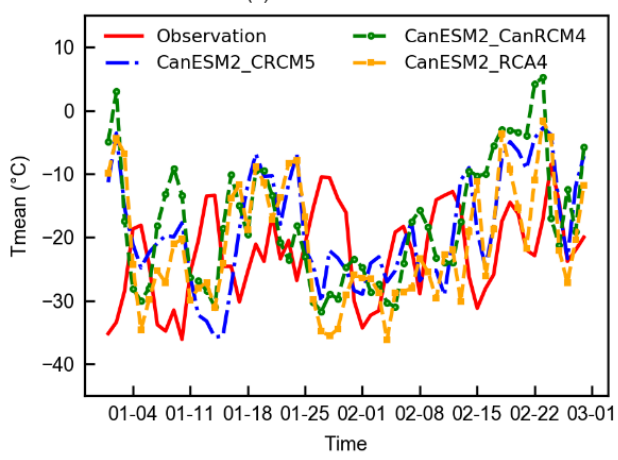

(b) RCM models winter
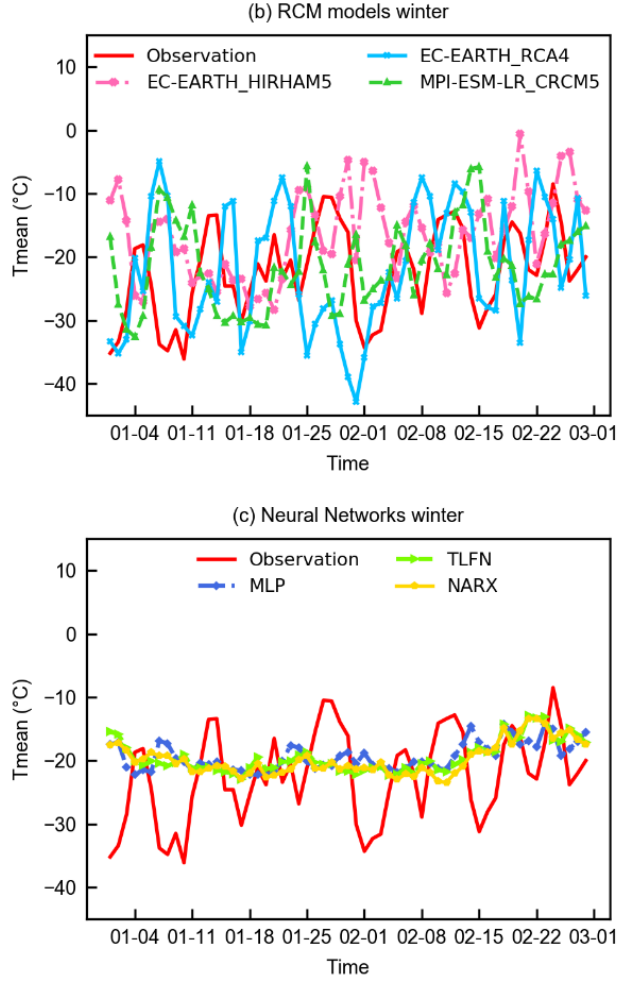

(d) RCM models summer

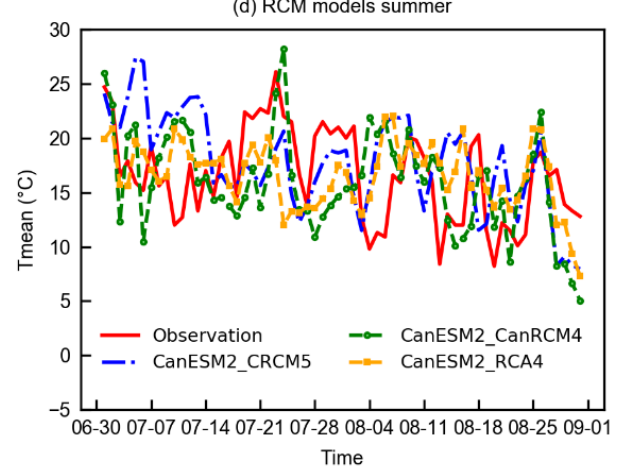

(e) RCM models summer
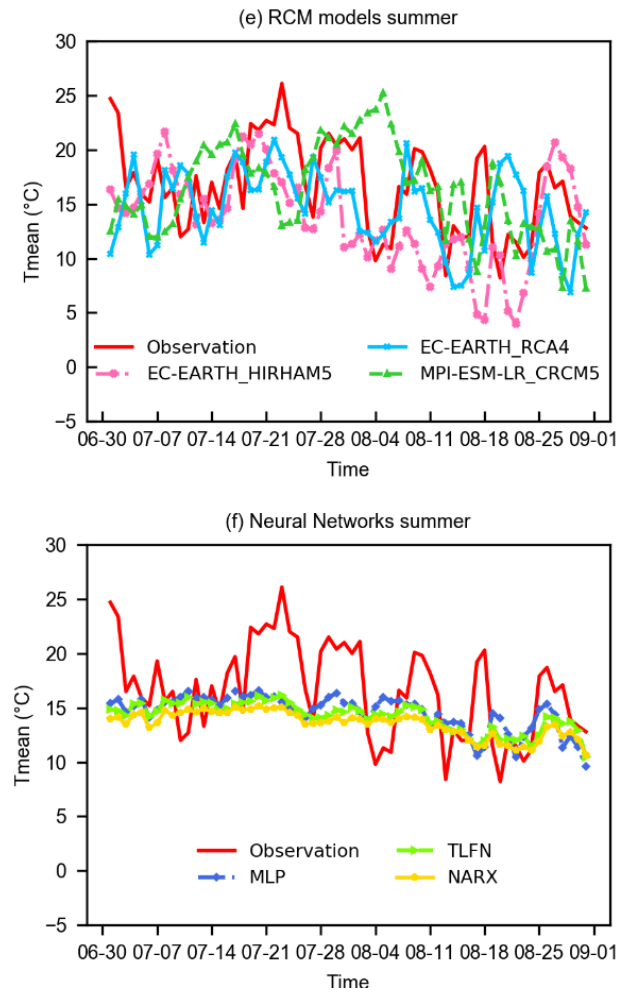

Figure 10. Time series plot of 6 RCMs and neural networks of $(a \sim c)$ winter and $(d \sim f)$ summer in 1989. 


\section{Conclusions}

The study investigated the applicability of three neural networks (MLP, TLFN, and NARX) for daily mean nearsurface temperature prediction using NA-CORDEX simulation output. A case study of Big Trout Lake in Ontario, Canada was carried out to demonstrate the applicability and performance of the three models. Daily mean temperatures simulated by six RCMs from 1979 to 1989 were applied for training, validation, and testing. The temperature values predicted by MLP, TLFN, and NARX were compared with the observations from the Big Trout Lake monitoring station. The performance of neural network models was compared with six individual RCMs.

The results show that MLP, TLFN, and NARX are effective methods for predicting daily mean temperature. Based on the RMSE and $\mathrm{R}^{2}$, all three methods had similar performance, with RMSE ranged from 6.345 to $6.537{ }^{\circ} \mathrm{C}$ and $\mathrm{R}^{2}$ above 0.84 . It is worth mentioning that the differences in prediction performance among these three models were not significant in terms of RMSE and $\mathrm{R}^{2}$. Neural-network based temperature prediction models outperformed individual RCM, with RMSE decreased by about $2{ }^{\circ} \mathrm{C}$ and $\mathrm{R}^{2}$ increased from 0.7 to 0.85 . Neural network models generated smoother predictions with less fluctuation than RCMs. It was also found that MLP, TLFN, and NARX could not capture 'extreme' values below $-20{ }^{\circ} \mathrm{C}$ accurately. Those values appeared during a similar time period each year (i.e., winter). Thus, further work could be done to develop prediction models for a seasonal time period which have different temperature range.

Acknowledgements. This research was supported by the National Key Research and Development Plan (2016YFA0601502).

\section{References}

Barfus, K. and Bernhofer, C. (2014). Assessment of GCM performances for the Arabian Peninsula, Brazil, and Ukraine and indications of regional climate change. Environmental Earth Sciences, 72(12), 4689-4703. https://doi.org/10.1007/s12665-014- 3147-3

Bush, E. and Lemmen, D.S., editors (2019): Canada's Changing Climate Report; Government of Canada, Ottawa, ON. PP. 444. https://changingclimate.ca/CCCR2019

Caswell, J.M. (2014). A Nonlinear Autoregressive Approach to Statistical Prediction of Disturbance Storm Time Geomagnetic Fluctuations Using Solar Data. Journal of Signal and Information Processing, 05(02), 42-53. https://doi.org/10.4236/jsip.2014.52007

Chan, R.W., Yuen, J.K., Lee, E.W., and Arashpour, M. (2015). Application of Nonlinear-Autoregressive-Exogenous model to predict the hysteretic behaviour of passive control systems. Engineering Structures, 85, 1-10. https://doi.org/10.1016/j.engstruct.2014.12.007

Çoruh, S., Geyikçi, F., Kılıç, E., and Çoruh, U. (2014). The use of NARX neural network for modeling of adsorption of zinc ions using activated almond shell as a potential biosorbent. Bioresource Technology, 151, 406-410. https://doi.org/10.1016/j.biortech.2013. 10.019

Coulibaly, P. (2004). Downscaling daily extreme temperatures with genetic programming. Geophysical Research Letters, 31(16). https: //doi.org/10.1029/2004GL020075

Coulibaly, P., Anctil, F., Aravena, R., and Bobée, B. (2001). Artificial neural network modeling of water table depth fluctuations. Water
Resources Research, 37(4), 885-896. https://doi.org/10.1029/2000 WR900368

Coulibaly, P., Anctil, F., and Bobee, B.J.J.o.H.E. (2001). Multivariate reservoir inflow forecasting using temporal neural networks. Journal of Hydrologic Engineering, 6(5), 367-376. https://doi.org/ 10.1061/(ASCE)1084-0699(2001)6:5(367)

Coulibaly, P., Dibike, Y.B., and Anctil, F. (2005). Downscaling Precipitation and Temperature with Temporal Neural Networks. Journal of Hydrometeorology, 6(4), 483-496. https://doi.org/10.1175/JHM 409.1

Dhussa, A.K., Sambi, S.S., Kumar, S., Kumar, S., and Kumar, S. (2014). Nonlinear Autoregressive Exogenous modeling of a large anaerobic digester producing biogas from cattle waste. Bioresource Technology, 170, 342-349. https://doi.org/10.1016/j.biortech.2014. 07.078

Diaconescu, E. (2008). The use of NARX neural networks to predict chaotic time series. Wseas Transactions on Computer Research, 3(3), 182-191.

Dibike, Y.B. and Coulibaly, P. (2006). Temporal neural networks for downscaling climate variability and extremes. Neural Networks, 19(2), 135-144. https://doi.org/10.1016/j.neunet.2006.01.003

Dibike, Y.B., Solomatine, D., and Abbott, M.B. (1999). On the encapsulation of numerical-hydraulic models in artificial neural network. Journal of Hydraulic Research, 37(2), 147-161. https://doi.org/10. 1080/00221689 909498303

ECCC. Digital Archive of Canadian Climatological Data. http:// climate.weather.gc.ca/historical_data/search_historic_data_e.html

Giorgi, F., Jones, C., and Asrar, G.R. (2009). Addressing climate information needs at the regional level: the CORDEX framework. World Meteorological Organization (WMO) Bulletin, 58(3), 175.

Hagan, M.T., and Menhaj, M.B. (1994). Training feedforward networks with the Marquardt algorithm. IEEE Transactions on Neural Networks, 5(6), 989-993. https://doi.org/10.1109/72.329 697

Hammerstorm, D. (1993). Working with neural networks. IEEE Spectrum, 46-53. https://doi.org/10.1109/6.222230

Haykin, S. (1998). Neural Networks: A Comprehensive Foundation: Prentice Hall PTR

Huo, A., and Li, H. (2012). Assessment of climate change impact on the stream-flow in a typical debris flow watershed of Jianzhuangcuan catchment in Shaanxi Province, China. Environmental Earth Sciences, 69(6), 1931-1938. https://doi.org/10.1007/s12665-0122025-0

Jiang, W., He, G., Long, T., Ni, Y., Liu, H., Peng, Y., Lv, K., and Wang, G. (2018). Multilayer perceptron neural network for surface water extraction in Landsat 8 OLI satellite images. Remote Sensing, 10(5), 755. https://doi.org/10.33 90/rs10050755

Karl, T.R., Melillo, J.M., and Peterson, T.C. (2009). Global Climate Change Impacts in the United States. Cambridge University Press.

Kumar, A., Mitra, A.K., Bohra, A.K., Iyengar, G.R., and Durai, V.R. (2012). Multimodel ensemble (MME) prediction of rainfall using neural networks during monsoon season in India. Meteorological Applications, 19(2), 161-169. https://doi.org/10.1002/met.254

LeCun, Y., Bengio, Y., and Hinton, G. (2015). Deep learning. Nature, 521(7553), 436-444. https://doi.org/10.1038/nature14539

Lee, C.C., Sheridan, S.C., Barnes, B.B., Hu, C., Pirhalla, D.E., Ransibrahmanakul, V., and Shein, K. (2016). The development of a non-linear autoregressive model with exogenous input (NARX) to model climate-water clarity relationships: reconstructing a historical water clarity index for the coastal waters of the southeastern USA. Theoretical and Applied Climatology, 130(1-2), 557-569. https://doi.org/10.1007/s00704-016-1906-7

Li, Z., Huang, G.H., Wang, X.Q., Han, J.C., and Fan, Y.R. (2016). Impacts of future climate change on river discharge based on hydrological inference: A case study of the Grand River Watershed in Ontario, Canada. Science of the Total Environment, 548, 198-210. https://doi.org/10.1016/j.scitotenv.2016.01.002 
Lin, T., Horne, B.G., and Giles, C.L. (1998). How embedded memory in recurrent neural network architectures helps learning long-term temporal dependencies. Neural Networks, 11(5), 861-868. https:// doi.org/10.1016/S0893-6080(98)00018-5

Lucas-Picher, P., Somot, S., Déqué, M., Decharme, B., and Alias, A. (2012). Evaluation of the regional climate model ALADIN to simulate the climate over North America in the CORDEX framework. Climate Dynamics, 41(5-6), 1117-1137. https://doi.org/10. 1007/s00382-012-1613-8

Mearns, L., McGinnis, S.; Korytina, D., Arritt, R., Biner, S., Bukovsky, M., Chang, H., Christensen, O., Herzmann, D., Jiao, Y.J., Kharin, Sl., Lazare, M., Nikulin, G., Qian, M.W., Scinocca, J., Winger, K., Castro, C., Frigon, A. and Gutowski, W. (2017). The NA-CORDEX dataset, version 1.0. NCAR Climate Data Gateway, Boulder CO. https://doi.org/10.5065/D6SJ1JCH

Osman, Y.Z. and Abdellatif, M.E. (2016). Improving accuracy of downscaling rainfall by combining predictions of different statistical downscale models. Water Science, 30(2), 61-75. https://doi. org/10.1016/j.wsj.2016.10.002

Palmer, T. N., Doblas-Reyes, F. J., Hagedorn, R., and Weisheimer, A. (2005). Probabilistic prediction of climate using multi-model ensembles: from basics to applications. Philosophical Transactions of the Royal Society B: Biological Sciences, 360(1463), 1991-1998. https://doi.org/10.1098/rstb. 2005.1750

Parmesan, C., and Yohe, G. (2003). A globally coherent fingerprint of climate change impacts across natural systems. Nature, 421(6918), 37-42. https://doi.org/10.1038/nature01286

Ragone, F., Lucarini, V., and Lunkeit, F. (2015). A new framework for climate sensitivity and prediction: a modelling perspective. Climate Dynamics, 46(5-6), 1459-1471. https://doi.org/10.1007/s00382-015 $-2657-3$

Rahimi, Z., Shafri, H., and Norman, M. (2018). A GNSS-based weather forecasting approach using Nonlinear Auto Regressive Approach with Exogenous Input (NARX). Journal of Atmospheric and Solar-Terrestrial Physics, 178, 74-84. https://doi.org/10.101 6/j.jastp.2018.06.011

Sfetsos, A., and Coonick, A.H. (2000). Univariate and multivariate forecasting of hourly solar radiation with artificial intelligence techniques. Solar Energy, 68(2), 169-178. https://doi.org/10.10 16/S0038-092X(99)00064-X

Shen, H.Y. and Chang, L.C. (2013). Online multistep-ahead inundation depth forecasts by recurrent NARX networks. Hydrology and Earth System Sciences, 17(3), 935-945. https://doi.org/10.5194/ hess-17-935-2013

Siegelmann, H.T., Horne, B.G., and Giles, C.L. (1997). Computational capabilities of recurrent NARX neural networks. IEEE Transactions on Systems, Man, and Cybernetics, Part B (Cybernetics), 27(2), 208-215. https://doi.org/10.1109/3477.558 801

Spak, S., Holloway, T., Lynn, B., and Goldberg, R. (2007). A comparison of statistical and dynamical downscaling for surface temperature in North America. Journal of Geophysical Research, 112(D8). https://doi.org/10.1029/2005JD006712

Spak, S., Holloway, T., Lynn, B., and Goldberg, R. (2018). The fate of Hudson Bay lowlands palsas in a changing climate. Arctic, Antarctic, and Alpine Research, 46(1), 114-120. https://doi.org/10.1657/1938424 6-46.1.114

Thomson, M.C., Doblas-Reyes, F.J., Mason, S.J., Hagedorn, R., Connor, S.J., Phindela, T., Morse, A.P., and Palmer, T.N. (2006). Malaria early warnings based on seasonal climate forecasts from multi-model ensembles. Nature, 439(7076), 576-579. https://doi, org/10.1038/nature04503

Lin, T., Horne, B.G., Tino, P., and Giles, C.L. (1996). Learning longterm dependencies in NARX recurrent neural networks. IEEE Transactions on Neural Networks, 7(6), 1329-1338. https://doi.org $/ 10.1109 / 72.548162$

Von Storch, H. (2000). Review of empirical downscaling techniques. In Regional climate development under global warming. General technical report no. 4. Conference proceedings regclim spring meeting Jevnaker, Torbjornrud, Norway, 2000.

Wagner, T., Themeßl, M., Schüppel, A., Gobiet, A., Stigler, H., and Birk, S (2017). Impacts of climate change on stream flow and hydro power generation in the Alpine region. Environmental Earth Sciences, 76(1), 4. https://doi.org/10.1007/s12665-016-6318-6

Wilby, R.L. and Wigley, T.M.L. (1997). Downscaling general circulation model output: a review of methods and limitations. Progress in Physical Geography: Earth and Environment, 21(4), 530-548. https://doi.org/10.1177/030913339702100403

Wilby, R.L., Wigley, T.M.L., Conway, D., Jones, P.D., Hewitson, B.C., Main, J., and Wilks, D.S. (1998). Statistical downscaling of general circulation model output: A comparison of methods. Water Resources Research, 34(11), 2995-3008. https://doi.org/10.1029/98 WR02577

Xia, Y., Chen, B., Weng, S., Ni, Y.Q., and Xu, Y.L. (2012). Temperature effect on vibration properties of civil structures: a literature review and case studies. Journal of Civil Structural Health Monitoring, 2(1), 29-46. https://doi.org/10.1007/s13349-011-0015-7

Zhang, D., Lindholm, G., and Ratnaweera, H. (2018). Use long shortterm memory to enhance Internet of Things for combined sewer overflow monitoring. Journal of Hydrology, 556, 409-418. https:// doi.org/10.1016/j.jhydrol.2017.11.018 